\section{Case Reports in Nephrology and Dialysis}

Case Rep Nephrol Dial 2020;10:117-123

\begin{tabular}{l|l} 
DOI: $10.1159 / 000508784$ & (C) 2020 The Author(s)
\end{tabular}

Published online: October 13, 2020

Published by S. Karger AG, Basel www.karger.com/cnd

This article is licensed under the Creative Commons Attribution-NonCommercial 4.0 International License (CC BY-NC) (http://www.karger.com/Services/OpenAccessLicense). Usage and distribution for commercial purposes requires written permission.

\title{
Neuroblastoma Amplified Sequence Gene Mutations Inducing Acute Kidney and Liver Injury in an Adolescent Female
}

\author{
Roy Rafael Dayan ${ }^{a} \quad$ O.N. Ray Bignall II ${ }^{b, c}$ Sheryl Johnson ${ }^{d}$ \\ Francisco Flores $^{c}$ Oded Volovelsky ${ }^{c, ~ e}$ \\ aFaculty of Health Sciences, Ben-Gurion University of the Negev, Beer-Sheva, Israel; \\ bivision of Nephrology, Nationwide Children's Hospital, Columbus, OH, USA; \\ 'Division of Nephrology and Hypertension, Cincinnati Children's Hospital Medical Center, \\ Cincinnati, $\mathrm{OH}, \mathrm{USA}$; dDivision of Pathology, Cincinnati Children's Hospital Medical Center, \\ Cincinnati, OH, USA; ePediatric Nephrology Unit, Hadassah Hebrew University Medical \\ Center, Jerusalem, Israel
}

\section{Keywords}

Human genetics · Acute kidney injury · Acute liver failure

\begin{abstract}
Acute liver injury $(A L I)$ in children is a life-threatening event, and a definitive etiology can be identified in approximately $50 \%$ of cases. Neuroblastoma amplified sequence (NBAS) gene mutations have been associated with a broad phenotypic spectrum of this disease, ranging from recurrent episodes of fever-induced liver injuries to multiorgan involvement, including frequent infections as well as skeletal and immunological abnormalities. Here, we describe an adolescent female with a confirmed compound heterozygous NBAS gene mutation who presented with an episode of $A L I$ complicated by severe acute kidney injury (AKI). The kidney injury was most probably driven by an intrinsic insult, as noted by elevated neutrophil gelatinase-associated lipocalin levels and a kidney biopsy demonstrating severe tubular damage consistent with acute tubular necrosis. While the patient's liver function and mental status showed significant improvement with supportive care, recovery of kidney function was
\end{abstract}




\section{Case Reports in Nephrology and Dialysis}

Dayan et al.: NBAS Gene Mutations Inducing Acute Kidney Injury

delayed, and the patient required acute hemodialysis. We suggest a causative relation between the NBAS gene mutation and severe AKI.

(C) 2020 The Author(s)

Published by S. Karger AG, Basel

\section{Introduction}

The neuroblastoma amplified sequence (NBAS) gene product was first described as an essential component of the syntaxin-18 SNARE complex, which participates in retrograde intracellular vesicular transport [1]. Knockdown of the NBAS gene in HeLa cells demonstrated functional rather than structural Golgi failure manifested by redistribution of Golgi recycling proteins accompanied by a defect in protein glycosylation. In addition to its role in retrograde vesicular transport, NBAS gene products play an essential role in the control of gene expression by participating in the nonsense-mediated mRNA decay surveillance pathway [2]. These varied cellular functions of NBAS might explain the potentially wide phenotypic variation associated with its mutation.

Pediatric acute liver injury (ALI) can be a life-threatening emergency, wherein approximately $50 \%$ of cases, the etiology is unknown [3]. Mutations in NBAS were initially described in 33 Siberian Yakut patients from 30 families presenting with so-called SOPH syndrome, which includes short stature, optic nerve atrophy, and Pelger-Huët anomaly of granulocytes [4]. In 2015, several reports of NBAS gene abnormalities were published. Haack et al. [5] reported biallelic NBAS gene mutations as the cause of recurrent infantile ALI in a cohort of 11 individuals from 10 German families. Segarra et al. [6] broadened the recognized phenotypic variations by reporting two unrelated cases of biallelic NBAS mutations presenting with multisystem disease involving the liver, bone, connective tissue, and immune system. He also noted a characteristic phenotype including progeroid appearance, short stature, slender bones, epiphyseal dysplasia with multiple phalangeal pseudoepiphyses, and cervical instability as a result of small C1-C2 vertebrae. In a separate, prospective observational study of 14 patients from 13 families with proven biallelic NBAS gene mutations who suffered from recurrent and fever-dependent ALI, data demonstrated a positive therapeutic effect of early antipyretic therapy and the administration of high parenteral glucose and lipid therapies on ameliorating the course of liver disease [7]. One patient presented with acute kidney injury (AKI) during a liver crisis, but whether this particular kidney injury represented a primary insult or an insult secondary to ALI remains unclear.

Although NBAS gene mutations have been associated with a broad clinical spectrum of multiorgan dysfunction, their relationship to AKI has been unstudied. We report a case of severe intrinsic AKI associated with NBAS gene mutation that may represent a novel link between this mutation and kidney dysfunction.

\section{Case Report}

A 16-year-old female with a complicated past medical history inclusive of recurrent and self-resolving episodes of ALI since birth presented to the emergency department with a 2day history of right upper quadrant abdominal pain, vomiting, and altered mental status.

A detailed history, obtained from both parents, described chronic medical complexity with multiorgan system involvement. The patient, born at 26 weeks of gestation, was diagnosed with developmental delay, short stature, and learning disability. Due to failure to thrive, she was gastrostomy tube dependent until 11 years of age. She was being treated for 


\section{Case Reports in Nephrology and Dialysis}

Case Rep Nephrol Dial 2020;10:117-123

DOI: $10.1159 / 000508784$

(c) 2020 The Author(s). Published by S. Karger AG, Basel www.karger.com/cnd

Dayan et al.: NBAS Gene Mutations Inducing Acute Kidney Injury

polycystic ovarian syndrome (managed with norgestrel, ethinyl estradiol, metformin, and spironolactone), hypothyroidism (managed with levothyroxine), chronic constipation (managed with polyethylene glycol), and anxiety disorder (managed with sertraline). Since birth, she had experienced several self-resolving ALI episodes of unknown etiology, characterized by elevated hepatic enzymes. According to her parents, the last ALI episode was 10 years before, probably triggered by a febrile illness. Although these episodes required hospital admission, each resolved spontaneously without any apparent long-term complications. Several genetic and metabolic workups failed to establish a definitive etiology for the ALI. The parents denied exposure to potential hepatotoxic agents or a special diet. The patient's immunizations were up-to-date, and workup for immune deficiency was negative.

On admission, her vital signs were remarkable for hypertension $(134 / 100 \mathrm{~mm} \mathrm{Hg})$, tachycardia (pulse of 114 beats/min), and tachypnea (respiratory rate of 28 breaths/min). Her oxygen saturation was $99 \%$ on room air. She was normothermic (body temperature of $36.9^{\circ} \mathrm{C}$ ), her height was $147.3 \mathrm{~cm}$ (1st percentile), and her weight was $52.1 \mathrm{~kg}$ (36th percentile). On physical examination, she was found to be drowsy, weak, difficult to arouse, and slow to respond to questions. Also, unsteady gate and asterixis were noted. Her abdomen was soft, with slight tenderness to palpation over the right upper quadrant. There was no organomegaly or ascites, and the rest of the physical examination was unremarkable.

Initial laboratory evaluation included a white blood cell count of $25,600 / \mu \mathrm{L}$, hemoglobin of $10.5 \mathrm{~g} / \mathrm{dL}$, and a platelet count of $270,000 / \mathrm{mm}^{3}$. Serum chemistries showed normal electrolytes and serum albumin, transaminitis with aspartate transaminase $>15,000 \mathrm{U} / \mathrm{L}$ (reference 5-26 U/L) and alanine transaminase 13,799 U/L (reference 12-49 U/L), hyperbilirubinemia (total serum bilirubin of $2.9 \mathrm{mg} / \mathrm{dL}$ ), hyperammonemia of $107 \mu \mathrm{mol} / \mathrm{L}$ (reference $\leq 32 \mu \mathrm{mol} / \mathrm{L}$ ), elevated lipase levels of 1,381 U/L (reference 64-231 U/L), coagulopathy with an INR of 2.8 (reference 1.0-2.0), lactic acidosis with a lactate level of $8.2 \mathrm{mmol} / \mathrm{L}$ (reference $1.0-2.4 \mathrm{mmol} / \mathrm{L}$ ), $\mathrm{pH}$ of 7.29, and serum bicarbonate of $15 \mathrm{mEq} / \mathrm{L}$ (reference $17-31 \mathrm{mEq} / \mathrm{L}$ ). The patient's elevated serum creatinine of $2.7 \mathrm{mg} / \mathrm{dL}$, with an estimated glomerular filtration rate of $22 \mathrm{~mL} / \mathrm{min} / 1.73 \mathrm{~m}^{2}$ (Schwartz formula), was consistent with stage III AKI based on the Kidney Disease: Improving Global Outcomes (KDIGO) classification [8]. Urinalysis was positive for proteinuria and microscopic hematuria ( $>50$ red blood cells per high-power field) without signs of infection. Chest radiography and computed tomography of the abdomen and pelvis were unremarkable, excluding any inflammatory or obstructive process. The toxicology screen was found to be negative as well.

While treated empirically with broad-spectrum parenteral antibiotics (piperacillin-tazobactam) and intravenous fluids, the patient's mental status improved, but her serum chemistries continued to worsen, prompting transfer to the pediatric intensive care unit with a diagnosis of ALI complicated by stage III AKI. On her second day of admission, preliminary infectious workup (including aerobic blood cultures, polymerase chain reaction, and serology for common viral etiologies) returned positive for influenza A infection, although she did not have fever, rhinorrhea, or any upper respiratory tract symptoms. Further diagnostic evaluation included acylcarnitine, urine organic acid, and amino acid profiles, all of which were within the normal range. To determine the etiology of the patient's ALI, she underwent a transjugular liver biopsy that revealed submassive hepatocellular necrosis with microvascular steatosis, suggesting metabolic rather than autoimmune etiology (Fig. 1).

Although encephalopathy, hyperammonemia, and overall liver function improved with conservative medical management, the patient's renal function continued to deteriorate. Despite an adequate urine output, her serum blood urea nitrogen and creatinine continued to worsen ( 44 and $4.8 \mathrm{mg} / \mathrm{dL}$, respectively), with an estimated glomerular filtration rate of 
$13 \mathrm{~mL} / \mathrm{min} / 1.73 \mathrm{~m}^{2}$. Due to the development of $5 \%$ fluid overload with pulmonary congestion and a new oxygen requirement, acute intermittent hemodialysis was initiated. An intrinsic kidney insult was suggested by urinary fractional excretion of sodium of $6.1 \%$ and elevated neutrophil gelatinase-associated lipocalin levels of 4,182 ng/mL (reference $<50 \mathrm{ng} / \mathrm{mL}$ ). Histology obtained by kidney biopsy demonstrated tubular injury with extensive tubular dilation, tubular epithelial necrosis, and glomerular sparing, most consistent with acute tubular necrosis (Fig. 2).

During the patient's hospital course, while liver function resolved almost wholly, renal recovery was delayed until 1 week after hospital discharge. In the setting of recurrent episodes of ALI and new-onset AKI, exome sequencing analysis confirmed a biallelic abnormality in the NBAS gene that could retrospectively explain her convoluted liver history and perhaps this recent episode of kidney involvement as well.

\section{Discussion}

The patient, in our case, was admitted to the pediatric intensive care unit with an episode of ALI characterized by hepatic encephalopathy and profound hepatocellular damage, complicated by severe AKI. In contrast to earlier events triggered by fever, she was afebrile throughout this ALI episode. Exome sequencing was performed and identified biallelic mutations in the NBAS gene, establishing a molecular basis for her recurrent episodes of ALI.

Different etiologies have been proposed to explain her renal injury in the setting of ALI. Though possible, functional AKI (formerly "prerenal azotemia") was less likely in the setting of hemodynamic stability with normal cardiac function, normal serum albumin levels, normal fluid status, elevated urinary fractional excretion of sodium, and an elevated urine neutrophil gelatinase-associated lipocalin level. Furthermore, kidney injury related to her liver injury (hepatorenal mechanism) was unlikely since her renal function continued to deteriorate long after her liver function had normalized, and she maintained a standard urine output. Abdominal computed tomography and renal sonography suggested against obstructive (postrenal) causes as well. These findings, together with a renal biopsy consistent with acute tubular necrosis, were strongly suggestive of an intrinsic renal insult associated with NBAS gene mutation.

The exact molecular mechanism in which the NBAS gene mutation might cause intrinsic AKI is not well recognized. Based on the understanding that these mutations simulate a "Golgi apparatus failure" state, one might hypothesize that the kidney injury pathogenicity resembles that of aminoglycoside-mediated nephrotoxicity. Aminoglycosides mediate renal injury by preferentially accumulating in the proximal renal tubule cells and poisoning the Golgi complex, endoplasmic reticulum, and lysosomal axis, which are responsible for protein sorting and synthesis [9]. Perhaps the NBAS gene mutations are similar to aminoglycoside toxicity in that both exhibit dysfunctional subcellular organelles state.

Although a causative relationship between NBAS gene mutations and severe AKI is impossible to deduce from a single case presentation, this novel association is intriguing and might be of great clinical significance.

In summary, we report the case of an adolescent female with a confirmed compound heterozygote NBAS gene mutation who presented with an episode of ALI complicated by severe AKI. To our knowledge, this is the first report of intrinsic AKI associated with NBAS gene mutation. Clinicians should be aware that under these clinical circumstances, intrinsic kidney injury is possible and might be severe enough to require renal replacement therapy until renal 


\section{Case Reports in Nephrology and Dialysis}

function is recovered. Additional investigation is necessary to further characterize this potentially meaningful relationship.

\section{Statement of Ethics}

This analysis was retrospective and a case report was not planned during routine clinical care. Though every conceivable effort has been made, the patient described in our case presentation was lost to follow-up, and no written informed consent is currently available. Nevertheless, our paper (1) does not contain any personally identifiable information, telling details, or recognizing data, (2) did not satisfy expectations for institutional review board submission at Cincinnati Children's Hospital Medical Center, and (3) was conducted in accordance with the World Medical Association Declaration of Helsinki.

\section{Conflict of Interest Statement}

The authors have no conflicts of interest to declare.

\section{Funding Sources}

The authors have no sources of funding to declare for this paper.

\section{Author Contributions}

All authors contributed equally to the literature review and the text of the manuscript. O. Volovelsky, O.N.R. Bignall II, and F. Flores were responsible for patient care and treatment.

\section{References}

1 Aoki T, Ichimura S, Itoh A, Kuramoto M, Shinkawa T, Isobe T, et al. Identification of the neuroblastomaamplified gene product as a component of the syntaxin 18 complex implicated in Golgi-to-endoplasmic reticulum retrograde transport. Mol Biol Cell. 2009 Jun;20(11):2639-49.

2 Longman D, Hug N, Keith M, Anastasaki C, Patton EE, Grimes G, et al. DHX34 and NBAS form part of an autoregulatory NMD circuit that regulates endogenous RNA targets in human cells, zebrafish and Caenorhabditis elegans. Nucleic Acids Res. 2013 Sep;41(17):8319-31.

3 Squires RH Jr, Shneider BL, Bucuvalas J, Alonso E, Sokol RJ, Narkewicz MR, et al. Acute liver failure in children: the first 348 patients in the pediatric acute liver failure study group. J Pediatr. 2006 May;148(5): 652-8.

4 Maksimova N, Hara K, Nikolaeva I, Chun-Feng T, Usui T, Takagi M, et al. Neuroblastoma amplified sequence gene is associated with a novel short stature syndrome characterised by optic nerve atrophy and PelgerHuët anomaly. J Med Genet. 2010 Aug;47(8):538-48.

5 Haack TB, Staufner C, Köpke MG, Straub BK, Kölker S, Thiel C, et al. Biallelic Mutations in NBAS Cause Recurrent Acute Liver Failure with Onset in Infancy. Am J Hum Genet. 2015 Jul;97(1):163-9.

6 Segarra NG, Ballhausen D, Crawford H, Perreau M, Campos-Xavier B, van Spaendonck-Zwarts K, et al. NBAS mutations cause a multisystem disorder involving bone, connective tissue, liver, immune system, and retina. Am J Med Genet A. 2015 Dec;167A(12):2902-12.

7 Staufner C, Haack TB, Köpke MG, Straub BK, Kölker S, Thiel C, et al. Recurrent acute liver failure due to NBAS deficiency: phenotypic spectrum, disease mechanisms, and therapeutic concepts. J Inherit Metab Dis. 2016 Jan;39(1):3-16. 


\section{Case Reports in Nephrology and Dialysis}

8 Kidney Disease: Improving Global Outcomes (KDIGO) Acute Kidney Injury Work Group. KDIGO Clinical Practice Guideline for Acute Kidney Injury. Kidney Int Suppl. 2012;2:1-138.

9 Sandoval R, Leiser J, Molitoris BA. Aminoglycoside antibiotics traffic to the Golgi complex in LLC-PK1 cells. J Am Soc Nephrol. 1998 Feb;9(2):167-74.

R.R. Dayan and O.N.R. Bignall II are co-first authors.

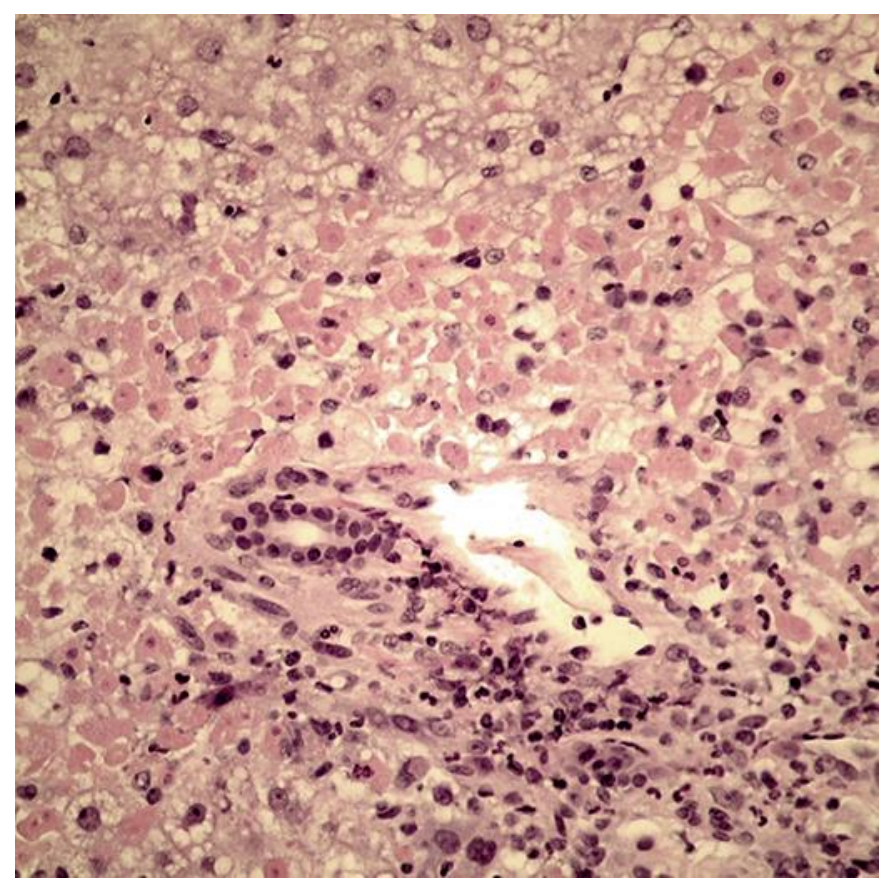

Fig. 1. Liver biopsy with hematoxylin-eosin staining demonstrates a portal tract that is expanded by a mixed inflammatory infiltrate comprised predominantly of lymphocytes and neutrophils. The portal tract structures are intact. Zone 1 shows marked necrosis, with more viable hepatocytes in zone 2 and 3. Microvesicular steatosis is conspicuous, particularly in the viable hepatocytes. 


\section{Case Reports in Nephrology and Dialysis}

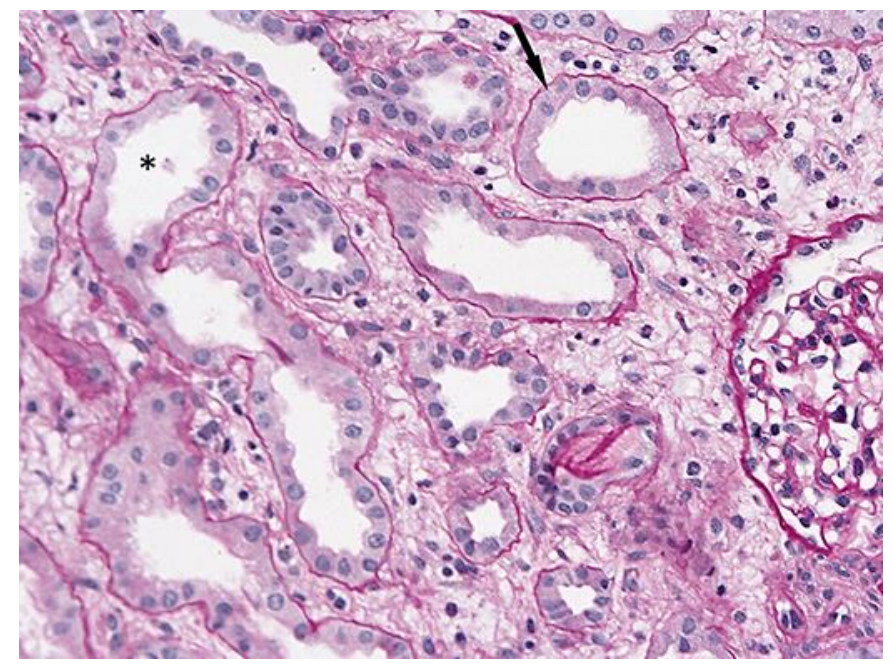

Fig. 2. Kidney biopsy with periodic acid-Schiff staining shows proximal tubules with significant dilation (asterisk), diminished brush border, and presence of intracellular debris. The tubular epithelium is flattened and filled with cytoplasmic vacuoles (arrow). 